\title{
CD47 Antagonist ALX148
}

National Cancer Institute

\section{Source}

National Cancer Institute. CD47 Antagonist ALX148. NCI Thesaurus. Code C132851.

A variant of signal regulatory protein alpha (SIRPa) that antagonizes the human cell surface antigen CD47, with potential phagocytosis-inducing, immunostimulating and antineoplastic activities. Upon administration, ALX148 binds to CD47 expressed on tumor cells and prevents the interaction of CD47 with its lig and SIRPa, a protein expressed on phagocytic cells. This prevents CD47/SIRPa-mediated signaling and abrog ates the CD47/SIRPa-mediated inhibition of phagocytosis. This induces pro-phagocytic signaling mediated by the binding of the pro-phagocytic signal calreticulin (CRT), which is specifically expressed on the surface of tumor cells, to low-density lipoprotein (LDL) receptor-related protein (LRP), expressed on macrophages. This results in macrophage activation and the specific phagocytosis of tumor cells. In addition, blocking CD47 signaling activates both an anti-tumor cytotoxic T-lymphocyte $(C T L)$ immune response and $T$ cell-mediated killing of CD47-expressing tumor cells. CD47, also called integrinassociated protein (IAP), is a tumor-associated antigen (TAA) expressed on normal, healthy hematopoietic stem cells (HSC) and overexpressed on the surface of a variety of cancer cells. Expression of CD47, and its interaction with SIRPa, leads to the inhibition of macrophage activation and protects cancer cells from phagocytosis, which allows cancer cells to proliferate. 